\title{
Smart Mask - Wearable IoT Solution for Improved Protection and Personal Health
}

\author{
Jarkko Hyysalo, Sandun Dasanayake, Jari Hannu, Christian Schuss, Mikko Rajanen, Teemu \\ Leppänen, David Doermann and Jaakko Sauvola
}

\begin{abstract}
The use of face masks is an important way to fight the COVID-19 pandemic. In this paper, we envision the Smart Mask, an IoT supported platform and ecosystem aiming to prevent and control the spreading of COVID-19 and other respiratory viruses. The integration of sensing, materials, AI, wireless, IoT, and software will help gathering of health data and health-related event detection in real time from the user as well as from their environment. In larger scale, with the help of AI-based analysis for health data it is possible to predict and decrease medical costs with accurate diagnoses and treatment plans, where comparison of personal data to large-scale public data enables drawing up a personal health trajectory, for example. Key research problems for smart respiratory protective equipment are identified in addition to future research directions.
\end{abstract}

Index Terms-COVID-19, edge computing, IoT, personal health, wearable.

\section{INTRODUCTION}

$\mathrm{T}$ HE worldwide public health emergency created by the coronavirus epidemic is continuing more than a year after the first cases were identified. Despite the availability of several types of vaccines, nonpharmaceutical interventions, such as social distancing and hand hygiene, play a significant role in mitigating the pandemic. Furthermore, the use of face masks is a way to prevent the transmission of COVID-19 infection as well as other respiratory viruses. Theoretical, experimental, and clinical evidence already suggested that face masks in the general population offer significant benefits in preventing the spread of respiratory viruses, especially in pandemic situations [1].

Even though only a handful of countries recommended using the facemasks at the beginning of the pandemic, now it has become a norm all around the world as more and more governments request their citizens to wear face masks. Some countries have even made it mandatory and imposed fines on those who don't comply.

Wearing a mask is seen at least as important as other mitigation approaches, like social distancing and handwashing. Wearing a mask complements other measures by controlling the harm at the source. Masks have become important for

This work was supported in part by the Business Finland under Grant $35795 / 31 / 2020$.

Authors J. Hyysalo, S. Dasanayake, J. Hannu, C. Schuss, M. Rajanen, T. Leppanen and J. Sauvola are with the University of Oulu, Pentti Kaiteran katu 1, 90570 Oulu, Finland (email: \{firstname.lastname\}@oulu.fi) essential workers as well as the general public as many countries are trying to move into the normal conditions with precautions and restrictions, rather than using strict lockdowns seen at the beginning of the pandemic. [2]

As masks are predicted to be used globally everywhere, leading to a huge demand. In 2019 the overall respiratory protective equipment (RPE) market size was 6.81 billion USD, and the growth is expected to be at a Compound Annual Growth Rate (CAGR) of $4.6 \%$ from 2020 to 2026. The value projection in 2026 is 11.05 billion USD. COVID-19 epidemic has increased the demand for protective equipment, and the current stockpile seems to be inadequate. Thus, the World Health Organization (WHO) suggested various companies and governments increase the manufacturing of respiratory protective equipment by $40 \%$ to meet demand. In addition to daily consumer use, the air-purifying respirator market is expected to continue to grow in industrial use, as they are used widely in several industries to protect the workers against dust, smoke, fumes, vapors, mists, and silica particles, for example. Another significant growth area is the medical and healthcare segment, with a $20 \%$ revenue share in 2019. [3]

Asia Pacific market is anticipated to reach 2.55 billion USD by 2027, while Europe is anticipated to progress at a CAGR of $9.0 \%$, and for example, in Germany, a CAGR of $8.4 \%$ is expected by 2027 . The largest application segment being the medical segment, and the fastest-growing segment being the personal care segment [4].

As the respiratory protective equipment markets are growing steadily, there are attractive opportunities for all players. A demand for multipurpose respiratory protective equipment is identified [5].

Digitalization of masks brings personal safety to the connected IoT- and AI-era. ICT brings the safety of protective physical gear and a complete digital package of sensing, analytics, and online services - a new business category to the smart wearable market.

This paper presents the Smart Mask concept, digitalized wearable protection that will provide improved protection against infections spreading through aerosol or respiratory droplets with integrated real-time data analysis for improving the immediate safety of the users. In addition to protecting

Author D. Doermann is with the University at Buffalo, Buffalo, New York, United States (email: doermann@,buffalo.edu) 
users, the Smart Mask will collect health and health-related data of the user. This allows creating a personal health and wellbeing history. The cumulative data, in combination with advanced large-scale data analytics, offers new tools for predictive and preventive healthcare.

With Smart Mask, the integration of sensing, materials, AI, wireless, IoT, and software will offer unseen service values. This will help people to gather health data in real-time as well as data from their environment. With data mashups based on different external data sources, we can offer something that has not existed in value-added services markets. A significant part of the Smart Mask concept is the accompanying applications and services that can be provided, and those open several new business opportunities.

This paper is organized as follows. Section 2 presents related work, Section 3 presents the Smart Mask concept with the key research problems, Section 4 presents and discusses the results, and finally, Section 5 summarizes the work.

\section{BACKGROUND}

Using nose and mouth covers as a part of the sanitary practices goes back to early modern Europe. However, the wide use of face masks by medical professionals can be traced back to the late 19th century and early 20th century. While the masks were initially used by the medical professionals as a means to prevent germs from entering into the patients, influenza pandemic in 1918-1919 turned it into a way of protecting both patients and medical workers from infectious diseases. Using disposable surgical masks has begun in the 1930s and become the norm among medical practitioners since around the 1960s [6]. During 2002-2004 SARS pandemic, the places such as Hong Kong recorded high usage of masks ranged from $61.2 \%$ $90 \%$ and the subsequent studies showed that it is a helpful strategy to delay or contain the pandemic, or at least to reduce the rate of infections [7].

The usage of masks has become one of the main frontline defense mechanisms during the ongoing COVID-19 pandemic $[8,9]$. While there is no sufficient evidence that wearing a surgical mask alone can protect the wearer from respiratory infections, the research indicates that it could effectively prevent transmission of respiratory infections from symptomatic individuals $[10,11]$. On the other hand, N95 respirators are more advanced types of disposable masks and they have a better protection for the wearer compared to surgical masks as it has a better-fit, fluid resistance and efficient filtration mechanism [12]. However, the masks can be an effective protection mechanism when it is bundled with the other infection control equipment and measures [13]. COVID19 pandemic has created a huge demand for the masks and as a result of that there was a major shortage of the masks. Specially, the N95 respirators and surgical mask supply was initially not enough even to cover the medical staff. That led researchers to investigate mechanisms to improve the reusability of the disposable masks as well as to develop reusable masks with advanced protection capabilities [14-16].

Smart healthcare utilizes modern technologies such as the internet of things (IoT), artificial intelligence (AI), big data, and edge and cloud computing, to make digitalized healthcare more available, convenient, efficient and personalized $[17,18]$. At the same time, the of use wearable healthcare technologies have moved beyond the medical practitioners and are becoming increasingly popular among the general consumers as a means to improve the self-wellbeing $[19,20]$. Smart Masks, with various technical capabilities in addition to the usual functions of the mask, have been developed for different purposes over the years [21-23]. However, general consumer oriented smart masks were not common until recently. COVID-19 pandemic resulted in increased research on smart masks [24-26] as well as commercially available smart masks $[27,28]$.

While smart masks can be a mix of many different technologies, in general smart masks have the ability to achieve advanced protection capabilities by optimizing the various build materials, filtering mechanisms, and integrated sensors, and other technologies [24-28]. The monitoring and measuring functionalities can provide various feedback to the user while enabling other permitted third parties such as healthcare professionals to remotely monitor the situation [25]. In addition, one of the main benefits of smart masks are their reusability [26]. Hence, the smart masks not only provide a solution to the mask shortage during a pandemic, but also help to address the issue of increased plastic pollution due to very high use of single use plastics as part of personal protective equipment in such situations [29].

\section{SMART MASK OVERVIEW}

The Smart Mask is a personal protective face mask with reusable and interchangeable filters, wireless upgradeable sensing service with health cloud analytics. The main components of the Smart Mask are 1) a reusable face mask shell with replaceable filter pack and plug-in sensor module; 2) a sensing module with embedded IoT device core, rechargeable batteries, Bluetooth radio, and vital sensors; 3) a mobile application for users and 4) edge and cloud computing backend as a health AI portal with personal health data storage, AI analysis, alerts, and recommendations.

The key research problems for the main components are presented in Table I. The Smart Mask concept and the main components are discussed in more detail in the following subsections.

\section{A. Mask, Materials and Design}

Various mask designs were studied to identify the most suitable for wearing smart masks. The most used mask types for COVID-19 protection are single-use surgical masks and reusable fabric-based face masks for breathing protection. Adding the electronics to the mask requires space from the mask, but also it adds weight to the mask. In addition, the masks must be reusable, at least the electronics part. The tested concepts were single-use/washable fabric mask with electronics module and 3D-printed mask with embedded electronics. The 3D-printed mask with a changeable filter was chosen to be the basis for the smart mask prototype. 
TABLE I

THE KEY RESEARCH PROBLEMS FOR SMART MASK

\begin{tabular}{|c|c|c|c|}
\hline & $\begin{array}{l}\text { Key research } \\
\text { problem } 1\end{array}$ & $\begin{array}{l}\text { Key research } \\
\text { problem } 2\end{array}$ & $\begin{array}{l}\text { Key research } \\
\text { problem } 3\end{array}$ \\
\hline $\begin{array}{l}\text { Mask, materials } \\
\text { and design }\end{array}$ & $\begin{array}{l}\text { The required } \\
\text { space and the } \\
\text { added weight of } \\
\text { electronics }\end{array}$ & $\begin{array}{l}\text { Mask design } \\
\text { accounting for } \\
\text { the washable } \\
\text { materials that } \\
\text { enable reuse }\end{array}$ & $\begin{array}{l}\text { User comfort } \\
\text { and easy filter } \\
\text { replacement }\end{array}$ \\
\hline $\begin{array}{l}\text { Sensing and } \\
\text { communication }\end{array}$ & $\begin{array}{l}\text { The type of } \\
\text { sensor and } \\
\text { placement }\end{array}$ & $\begin{array}{l}\text { Real-time } \\
\text { communication } \\
\text { requirements }\end{array}$ & $\begin{array}{l}\text { Energy } \\
\text { management }\end{array}$ \\
\hline $\begin{array}{l}\text { Application and } \\
\text { UI }\end{array}$ & $\begin{array}{l}\text { User } \\
\text { requirements } \\
\text { for the Smart } \\
\text { Mask }\end{array}$ & $\begin{array}{l}\text { Maximizing the } \\
\text { level of user } \\
\text { acceptance }\end{array}$ & $\begin{array}{l}\text { Seamless } \\
\text { connection and } \\
\text { user experience }\end{array}$ \\
\hline $\begin{array}{l}\text { Backend and } \\
\text { health AI }\end{array}$ & $\begin{array}{l}\text { Safety of the } \\
\text { Smart Mask } \\
\text { user utilizing a) } \\
\text { mask sensor } \\
\text { data b) other } \\
\text { data }\end{array}$ & $\begin{array}{l}\text { Real-time } \\
\text { monitoring, } \\
\text { analyzing, and } \\
\text { managing the } \\
\text { platform }\end{array}$ & $\begin{array}{l}\text { Services for } \\
\text { long-term } \\
\text { health data } \\
\text { storage and big } \\
\text { data analytics }\end{array}$ \\
\hline
\end{tabular}

The mask prototype consists of a 3D-printed chassis with an easily removable module for electronics. The filter is replaceable, and the mask is attached to the head with stretchable, adjustable bands behind the user's head.

Also, cloth-based reusable masks and single-use surgical masks with embedded electronics were tested. The light masks are comfortable to use, but the added electronics module made the usage more uncomfortable. The module was too heavy without supporting structures and caused misalignment of the mask. In addition, the module would touch the face, which is not comfortable to the user and causes measurement error with sensors directly touching the user's face. The electronics module cannot be disposable; therefore, the electronics module must be attached to new masks, which again causes reliability issues regarding the airtightness of the mask.

The 3D-printed mask was printed with flexible filament FlexFill 98A (Fillamentum, Czech Republic). The filament is based on thermoplastic polyurethane (TPU), which is flexible compared to typical rigid polylactide (PLA) filaments used in 3D-printing. The flexible filament enables a more comfortable mask when used compared to rigid mask parts. The 3D-printed elements were designed with Solidworks. Fig. 1 presents the designed components for 3D-printing.

As can be seen from Fig. 1, the filter gains the first attention. The size of the filter was iterated a few rounds by testing various areas. Too small a filter area was uncomfortable, and the user was required to use extra force for breathing. The larger area filter was found to be best for most test users. The filter material itself is trapped between two 3D-printed holders, from where it is easy to change. The used filter material was fibrous polyester or polypropylene materials made by non-woven techniques. The materials are intended to be disposable. Washing impairs filtration. The efficiency of the material is largely based on static forces that trap small particles. The better the filtering capability is, the harder it is to breathe through, and for this reason, a larger filter area is used.
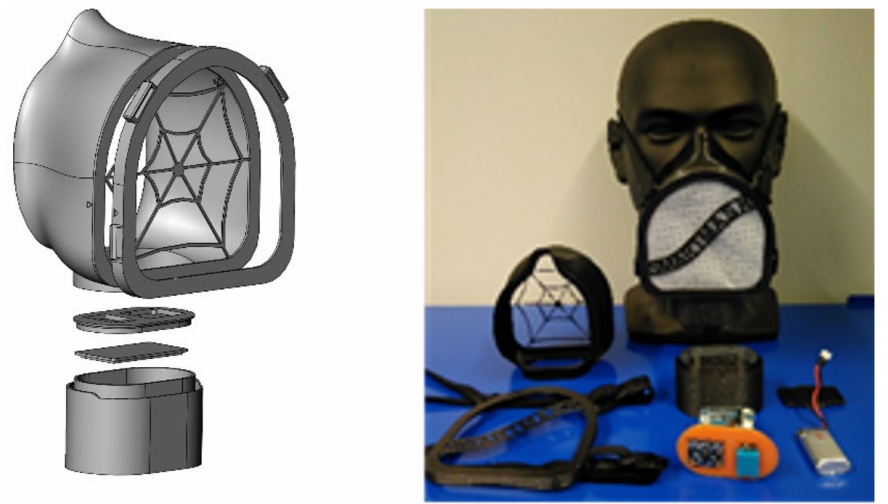

Fig. 1. a) Design image of the assembly of the 3D-printed mask. b) 3D-printed early prototype of the Smart Mask on the model head and disassembled version of the mask in front.

\section{B. Sensing and Communication Module}

The aim of the sensing and communication module is to provide users with accurate and reliable information, for example, about the air quality underneath the mask or possible movement of the mask. Hence, in this work, we concentrated on the types of sensors that need to be integrated into the prototype of the Smart Mask for obtaining this relevant information and data.

Different types of sensors can be used to detect certain types of events. As such, any movement of the mask can be detected in various ways. In principle, a mask that is not well attached to the user's skin provides limited protection. For example, ambient light sensors (ALS) can be placed underneath the mask to measure the light intensity. At a particular light intensity, in other words, when the available light exceeds a threshold value, it can be assumed that the mask does not sit well on the user's skin and, as a result, does not fully protect the user. Likewise, the information from an accelerometer can be utilized to measure the potential movement of the Smart Mask. Sensors can also be embedded in the holder of the mask and, by measuring changes in the resistance, it can be assumed that the mask has moved, and the user needs to readjust their mask.

In a similar way, the air quality underneath the mask can be measured with the help of an equivalent carbon dioxide $\left(\mathrm{eCO}_{2}\right)$ sensor, which can measure at the same time the number of total volatile compounds in the air. It is worth noting that if the air quality underneath the mask is degrading, it is more likely that users are touching their mask to relieve hot air from underneath. Here, if users touch their mask, they can transfer the virus onto it. For maximizing the user's level of protection, intentional or unintentional movement from the mask needs to be avoided by the user.

In general, sensors embedded into the Smart Mask need to provide users with accurate and reliable information. Thus, we studied the impact of the sensor location within the mask on sensor readings. As an example, when monitoring the temperature underneath the mask, we noticed that the placement of the sensor (e.g., at the button of the mask or on the side of the mask) affects the obtained temperature. As such, a temperature sensor provides higher temperature readings depending on the placement of the sensor in the mask.

As mentioned above, the air quality underneath the mask was one of the key parameters investigated during the design and 
development of the Smart Mask. It was found out that the temperature rises quickly once the user puts on their mask. However, after some time, the temperature starts to saturate if the user remains steady (e.g., when sitting in a bus). In this work, it was found out that the choice of the filter has an impact on the saturation temperature. It is worth noting that each user will have an individual comfort level and, hence, a different threshold temperature which is acceptable underneath the mask. As a rule of thumb, the bulkier the filter (i.e., the higher the protection from particles), the hotter the air underneath the mask can become.

Once data from sensors is obtained, it is crucial to provide the obtained sensor information to users immediately. In this way, we concentrated on real-time communication between the sensors and the user's smartphone. For the prototype of the Smart Mask, we utilized Wi-Fi communication. However, in the next version of the prototype, due to the required data rate and in terms of improving the energy efficiency of the Smart Mask, we consider using Bluetooth Low Energy (BLE) instead of Wi-Fi communication.

Energy management is an important aspect of the Smart Mask. The battery life is critical for user satisfaction. In other words, if users must recharge the battery of their mask often, they can get annoyed. Furthermore, the rechargeable battery of the mask is one of the major contributors to the weight of the mask. If the communication between the Smart Mask and the smartphone requires a lot of energy and/or if obtaining data from sensors is reducing the state of charge $(\mathrm{SoC})$ of the battery rapidly, then a larger battery capacity can be needed, increasing, therefore, the overall weight of the mask.

In summary, there are various aspects that need to be considered for providing users with a sophisticated and lightweighted Smart Mask design. Firstly, sensor information needs to be accurate and reliable. The type of sensor and placement has an impact on measurement results. Secondly, real-time communication ensures that users are instantly informed if there is an issue with their mask requiring attention. Finally, maximizing and optimizing the battery lifetime of the Smart Mask ensures user satisfaction and comfort of wearing the mask.

\section{Integrated Application and UI}

A mobile application was designed and developed to act as a companion to the physical Smart Mask. The application was designed using user-centered design in order to maximize the level of user acceptance and user experience and to identify user requirements, context requirements, and cultural aspects related to mask use and to provide seamless connection and user experience of Smart Mask, application, sensors, analytics and data visualizations between the mask, app, and backend.

The UI of the mobile application gives vital information about the status of the Smart Mask as well as alerting the user when needed (e.g., battery running low, an air leak in the mask, the filter needs to be replaced). These alerts raised within the Smart Mask ecosystem were a vital part of ensuring safe operating conditions. The user interface of the mobile application was based on user research conducted at the beginning of the project by in-depth interviews of seven potential users representing different user groups and contexts of use. Furthermore, the user research provided vital user requirements for the design of the physical Smart Mask as well as for the functionalities and usability of the Smart Mask mobile application. Based on these results, user interface concepts for the mobile application were designed and tested. Different anonymized user interface design concepts were tested in 75 user tests to map out user interface preferences and to test the clarity and understandability of the mobile application UI.

The main UI view shows the general status of the protection with appropriate colors (green, yellow, red) and status messages. Entering yellow (endangered) and red (compromised) states will result in a notification/alert being displayed on the phone screen, and an alarm signal is heard. Pressing the area around the "Note" icon will display contextdependent graphical instructions on adjusting the mask. The other views of the application include the protection view, mask air quality view, mask status view, and outside air quality view for external data. Pressing (or tapping) the protected area on the default application view will display information about the level of protection and instructions on how to adjust the mask for proper protection. The level of protection and the relevant instructions is displayed as long as the status is valid. The air quality view operates in a similar way, displaying information about the overall mask air quality and measurements of humidity, temperature, $\mathrm{CO}_{2}$, contaminants, and pollutants. The mask status view, on the other hand, displays more detailed information about the battery level, filter status, and connection status. In addition, the outside air quality using Air Quality Index scale (good, moderate, unhealthy for sensitive groups, unhealthy, very unhealthy, and hazardous) and the reported COVID-19 cases in the user's health service region can be reported.

The application was connected to an edge message broker relaying mask data from the mobile application to analysis components and alerts and notifications across the platform. Communication and the display of alerts were done in real-time for the currently selected mask. The application allows users to change the mask they are wearing, provides means for indicating a filter change, and provides a switch for indicating whether the mask is currently in use. For user testing, the application was remotely piloted by issuing commands through the message broker. This enabled the usability tester to carry out Wizard-of-Oz -type testing scenarios remotely during COVID-19 lockdown. The usability and user experience of the application were tested with five users representing different user groups and contexts of use. The results of these tests indicate that the application UI and UX were good. The intended user groups could use the application with effectiveness, efficiency, and good user experience.

\section{Backend and Health AI}

The role of the Health AI backend is to provide (1) seamless supporting data analytics services for the online smart mask operation, (2) facilitate collaboration between multiple users and stakeholders during the operation, (3) provide means for failure recovery, controlled sharing of data, external data sources and 3rd party service integration into the platform and (4) provide services for long-term health data storage and Big Data analytics aimed for a diverse set of stakeholders.

First, during the online operation, the safety of the smart mask user is the utmost concern. Therefore, in addition to the mask 
sensor data analysis, the user needs to be informed about possible mistakes in the mask usage and recommendations based on analysis of users' contextual use case-specific personalized health data. Moreover, informing the user about possible dangers due to mistakes, device failures, or changes in the operational environment, e.g., rapidly changing environmental parameters such as leaks of dangerous substance into the air, data analysis component is needed in operational premises to create real-time alerts for users.

Second, the platform should facilitate location-based monitoring of the masks and actions of multiple users in some local environments, such as hospital emergency rooms. The backend platform should integrate the mobile applications, sensor data collected from the masks, personalized health data, and location-based environmental data for in-premises realtime analytics to provide an aggregated view over the operation to facilitate safe collaboration and co-operation of users in such possibly hazardous environments.

Third, the platform itself should be able to monitor and analyze its own online operation and performance for increased protection and to recover from software and hardware component failures and network connectivity issues. Minimally, the users should be informed about changes in the operational state of the platform, network connectivity, and related risks.

Fourth, the Health AI component in the backend provides means for long-term data storage, collected from the masks and the operational environment, and for related analytics. The results of such analysis are beneficial for multiple stakeholders, such as the user, platform administration, 3rd party service providers, researchers, and public 3rd party service providers, to name a few.

Therefore, we adopted the foreseen edge computing paradigm for the smart mask platform and backend design and prototype implementation atop underlying IoT networks to which the smart masks and mobile applications for users are connected to. Edge computing enables harnessing the end-user devices (e.g., smartphones) and network infrastructure components, such as gateways, as a part of distributed computing platform where partitioned distributed IoT application components can be leveraged from the cloud through virtualization [30]. Virtualization in different scales allows a diverse set of options for the deployment of these software components into the underlying system and network. In this light, we design the backend functionality in a way that the real-time analysis components can be placed directly in the operational premises of the smart mask users. Further, we adopted current industry software development practices, i.e., DevOps, CI/CD, and microservices, as the platform component development solution. As such technologies are currently well-known and widely used, the integration of 3rd party services and external data sources becomes straightforward. Moreover, existing edge platform management solutions (e.g., Kubernetes and microservice virtualization with containers) have become available with existing capabilities for system monitoring and failure recovery.

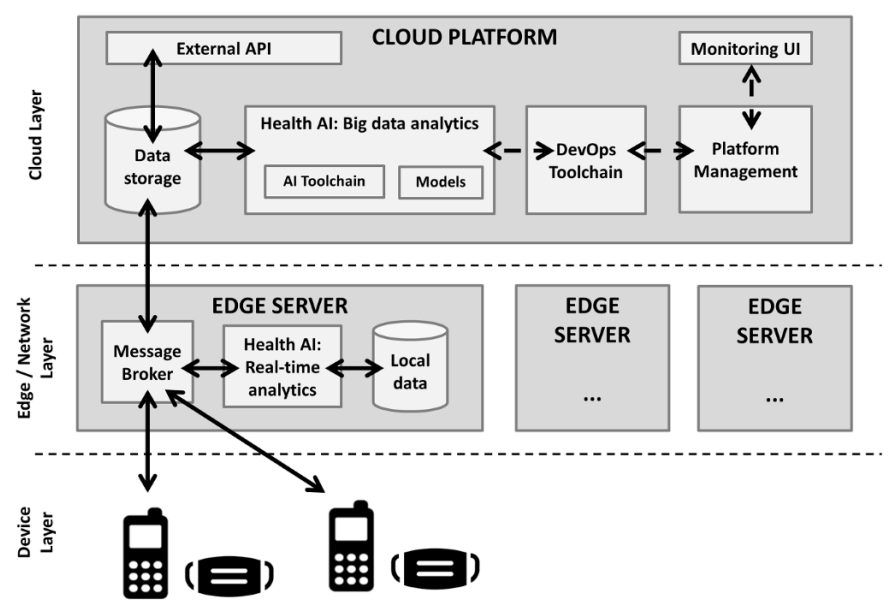

Fig. 2. Smart Mask architecture.

The resulting software architecture of the smart mask platform and backend is depicted in Fig. 2. Starting from the cloud layer, we place here the smart mask functionality software components as microservices, inherently building distributed application logic with varying deployment options based on their computational and networking resource requirement across the layers. Platform management solution, such as Kubernetes, is needed to semi-automatically run the platform, deploy software components and monitor their performance. Additionally, we designed a real-time monitoring UI for administrators to monitor the platform state, i.e., the state of the microservices, connected devices, network connectivity as an additional safety measure. For example, a monitoring microservice should collect data of the platform operation in real-time and visualize and send alerts to users based on realtime analysis of the data. The cloud layer also contains a database solution for long-term data storage for the mask sensor(s), user and operational environment data, and the exposed interface for external 3rd party service integration.

On the edge layer, we placed the data analysis component that creates information in real-time about the operational conditions in premises, where the mask users are. Integrated message broker then administrates the data and information delivery across the system, which placement close to the user facilitates accelerated delivery of the priority information. Brokered solution for message delivery supports different types of messages for data dissemination with different data types in multiple application scenarios and notifications (e.g., informative / alerts) and separate system control commands. Using a well-known solution, e.g., the MQTT(S) protocol, facilitates easy integration of additional software components across the layers.

On the device layer, we placed the Smart Mask and their mobile applications that are wirelessly connected to the onpremises edge server providing multiple users the real-time analysis services and message delivery.

\section{Results AND Discussion}

Currently, the mass market is for "unintelligent" cloth masks, respirators, and surgical masks. Only a few products exist that try to add intelligence to the mask. 
TABLE II

SMART MASK IN COMPARISON TO OTHER SOLUTIONS.

\begin{tabular}{|c|c|c|c|c|c|c|c|c|}
\hline & Smart Mask & $\begin{array}{c}\text { LG } \\
\text { PuriCare }^{\mathrm{TM}} \\
\end{array}$ & Razer & Forcit & AirPop & Cloth mask $^{*}$ & Respirator $^{* * *}$ & $\begin{array}{c}\begin{array}{c}\text { Surgical } \\
\text { mask }^{* *}\end{array} \\
\end{array}$ \\
\hline Medical use & & & & $\mathrm{X}$ & & & & $\mathrm{X}$ \\
\hline Comfort & $\mathrm{X}$ & $\mathrm{X}$ & $\mathrm{X}$ & $\mathrm{X}$ & $\mathrm{X}$ & & & \\
\hline \multicolumn{9}{|c|}{ Added electronic functionality } \\
\hline Sensing & $\mathrm{X}$ & & & & $\mathrm{X}$ & & & \\
\hline Ventilation & & $\mathrm{X}$ & $\mathrm{X}$ & & & & & \\
\hline \multicolumn{9}{|c|}{ Intelligence } \\
\hline $\begin{array}{l}\text { Real-time AI- } \\
\text { based guidance }\end{array}$ & $\mathrm{X}$ & & & $\mathrm{X}$ & $\mathrm{X}$ & & & \\
\hline $\begin{array}{l}\text { Population-level } \\
\text { advice }\end{array}$ & $\mathrm{X}$ & & & & & & & \\
\hline $\begin{array}{r}\text { Data mashup } \\
\text { and sharing }\end{array}$ & $\mathrm{X}$ & & & & & & & \\
\hline
\end{tabular}

*Multiple vendors including Razer \& DIY. **Multiple vendors including 3M, Honeywell, Kimberly-Clark, and Owens \& Minor.

The most advanced masks in the markets are "stand-alone" products with few extra features (e.g., fans that ease breathing through masks or from sensors that detect the air intake), not integrated and intelligent systems that are a part of a complete ecosystem, like Smart Mask (i.e., device+cloud+application).

Table II. compares Smart Mask to other alternatives. In Table II, filtration is the basic feature of the mask, its ability to filter unwanted substances. Medical use considers if the mask has a medical-grade. Style/fashion shows if it is possible to have different looks, or patterns for the mask, features making the mask more desirable. Comfort denotes that it is comfortable to wear. Added electronic functionalities, like sensing, considers if the mask has the ability to measure various factors. Ventilation means that there is a fan in the mask. Voice correction, in turn, improves/amplifies the speech. Intelligence features, like AI-based guidance studies, if the mask provides guidance, alerts and notifications for the user. Population-level advice denotes the ability to provide advice also for the wider public. A data mashup and sharing studies determine if the backend AI analysis can benefit from data from other sources and share the data and analysis results (in a safe, secure, and anonymized way).

The selection of other alternatives includes LG PuriCare ${ }^{\mathrm{TM}}$, Razer, and AirPop, which are all already available products or coming to markets in 2021. Forcit is a research project that is still ongoing, like Smart Mask, both having already prototypes for testing. Cloth masks, respirators, and surgical masks are non-smart masks, also included here for comparison purposes. The comparison was made based on data available on the vendor's webpages.

Our solution for smart mask, provides digitalized wearable protection. The Smart Mask will provide a completely new offering to markets aimed at customers willing to invest in customized safety. Smart Mask offers "intelligence as a service" and provides very personal health and wellbeing benefits. It is an N95 type of reusable respiratory mask, complemented with changeable sensors, real-time connections to backend analytics and user mobile application.
The smart mask solution prevents COVID-19 spread through normal breathing and speech. The Smart Mask and its ecosystem offer "AI as a service" and provide personal health and wellbeing benefits. The smart sensing module can be configured with various sensors measures such as $\mathrm{CO}_{2}$ expirations, pulse, temperature, and air intake. The Smart Mask can also measure air pollution and several other environmental variables. The measurements are stored on an IoT platform, where users can monitor their own health data with a mobile application.

Smart Mask will add features that are not available elsewhere, such as improved safety through sensing airtightness and the quality of filters. Measurements from changeable sensors and linking to backend allow direct personalized realtime data, which enables the creation of applications and services to improve health and healthcare, based on modern IoT software development paradigms. Data analysis (e.g., data mining and machine learning) is to be used to predict and decrease medical costs with accurate diagnoses and treatment plans. Sharing and mashing up external data, anonymized location, environmental data and related information as a service advances digitalized health IoT service concepts and creates a new level of safety. Comparison of individual data to large-scale health data repositories enables drawing up a personal health trajectory, for example.

The Smart Mask presents a tremendous opportunity to apply advanced analytics for healthcare. If the data is made available to analytics, the latest methods can be applied for both real-time and predictive use cases. When the data is shipped to the cloud, there are numerous possibilities available for applying advanced AI techniques, such as deep neural networks to model parameter norms and help classify outliers. Furthermore, longterm trends can be identified, or data can be associated with related populations under normal conditions.

We envision a pipeline that initially begins with rule-based alerts that statistically analyze incoming data streams to identify outliers based on an individual's history and local norms. As more data becomes available, we will be able to apply more 
sophisticated data-intensive algorithms. One of the challenges of machine learning is to have adaptive models that can change over time. They need to be able to change as the smart mask is used in different environments, at different times of the day, or even seasonally. This is an active area of research and is often addressed by applying different models for different temporal dependencies. In the cloud, all of the latest machine learning and data science tools are at our disposal.

One of the important considerations, however, is that these masks are truly edge devices. They gather data locally, and while we assume connectivity, there are a number of drivers that must be considered. If we assume real-time diagnostics, latency, the cost of a round-trip connection with the cloud, and connectivity that may not always be available are serious considerations. If we eventually add audio or video components to the smart mask, then bandwidth may also become a concern. The fourth concern is the heterogeneity of the data. Depending on the location, population, or even version of the device, the data may not be presented or recorded in an identical manner. This is typically taken care of by server-side data science algorithms but must be considered.

A final and significant challenge, however, is security and privacy. The basic level of security may include, for example, end-to-end encryption. Nevertheless, there are times when individuals either don't want to or can't share their data. This leads to important considerations for edge intelligence. Most edge computing today is focused on the concept of edge caching. This is the view of taking and providing data in a way that enhances the user experience by reducing the effect of the challenges described above. For data caching, an edge-aware system will attempt to predict what is required from the server and provided it to the user to reduce latency and delay challenges. Systems may also try to aggregate data locally before sending it to the server to reduce bandwidth. But in our application, this is of minimal concern. A real concern is a privacy.

Privacy concerns lead to the concept of edge training. Are we able to train our models locally, perhaps on the connected mobile device, reducing the need to share private information? The answer is general is yes, but in our case, the lack of a large amount of data makes it unlikely to succeed. What is really required is the ability to train a model with the input, but not necessarily the data, of individual masks. One solution is what is known as federated learning.

The concept of federated learning has a goal of training a high-quality model with a combination of central, publicly available data and private data from individual nodes that cannot be shared. Unlike standard approaches where the data comes together centrally, the learning environment needs to update a central model from the partial results of training locally at individual nodes with a combination of the public data and its own private data. A typical scenario for training maybe for the central system to provide some share data, and each node trains with this data and its local data. The local model surpassed back to the central server to be updated, and the new central model is distributed back to all of the individual nodes to be updated. This continues for a number of iterations until the system stabilizes. At this point, there's a central model that benefits all of the local nodes, but the individual nodes do not have access to other data, or do they share their data with the central model. Recently there have been a number of applications, particularly in the area of medical image analysis, that share only weights and local gradients during training. Nevertheless, the overhead for communications can be high, and there is a security risk if this information is exposed and aggregated across multiple systems.

Even with the ability to train models locally and contribute to a more centralized model, these edge devices typically have very limited resources. The question of how to provide edge inference is also a concern. The state-of-the-art in edge inference focuses on a pipeline where full precision models are compressed, pruned, or otherwise quantized to produce a model that is significantly less compute-intensive and physically smaller to fit these processing and memory-constrained devices. This will be especially useful when work masks add additional image and audio acquisition capabilities.

\section{CONClusion AND Future Work}

This paper presented a Smart Mask with accompanying ecosystem and intelligent services to prevent COVID-19 and other respiratory viruses. Some smart RPEs are already available that provide different features. People already demand "advanced masks" with no ICT, willing to pay up to hundreds of euros for "designer" masks. Users also want differentiation Masks are increasingly perceived as a fashion statement while staying safe - offerings can be branched to fashion, sports, elderly, clinical, and so on.

The Smart Mask is not just a one-off solution. Instead, it offers an innovative multi-disciplinary RDI venue. We aim for the interplay of scientific research, technology transfer, and global market opportunity with the continuous creation of RDI breakthroughs for smart wearables markets.

Differentiation comes via a combination of immersive sensing, AI, IoT, and wireless experience. Smart Mask is a total value proposal that brings the protective gear and services to the next level: augmented self-protection with health tracking and personalization. Smart Mask level-up the safety, selfexpression, online and real-time services for personal protection.

In addition, several avenues for future work have already been identified in the context of smart RPEs, such as: adaptive models for machine learning, latency, the cost of a round-trip connection with the cloud, connectivity, bandwidth, and especially security and privacy issues.

\section{REFERENCES}

1. Gupta, M., Gupta, K., and Gupta, S., "The use of facemasks by the general population to prevent transmission of Covid 19 infection: A systematic review," medRxiv, 2020,

https://doi.org/10.1101/2020.05.01.20087064

2. Cheng, K. K., Lam, T. H., and Leung, C. C., "Wearing face masks in the community during the COVID-19 pandemic: altruism and solidarity," The Lancet, 2020, https://doi.org/10.1016/S0140-6736(20)30918-1 
3. Pulidindi, A. and Pandey, H., "Respiratory Protective Equipment Market Size By Product," 2020. [Online]:

https://www.gminsights.com/industry-analysis/respiratory-protectiveequipment-market

4. Grand View Research, "Respiratory Protective Equipment Market Size, Share \& Trends Analysis Report By Product," 2020. [Online]: https://www.grandviewresearch.com/industry-analysis/respiratoryprotective-equipment-market

5. Markets and Markets, "Respiratory Protection Equipment Market by Product Type," 2017. [Online]:

https://www.marketsandmarkets.com/Market-Reports/respiratoryprotection-equipment-market-26333088.html

6. Strasser, B. J. and Schlich, T., "A history of the medical mask and the rise of throwaway culture," The Lancet, vol. 396, no. 10243, pp. 19-20, 2020. [Online]: https://doi.org/10.1016/S0140-6736(20)31207-1

7. Brienen, N. C., Timen, A., Wallinga, J., Van Steenbergen, J. E., and Teunis, P. F. (2010). The effect of mask use on the spread of influenza during a pandemic. Risk Analysis: An International Journal, vol. 30, no. 8, pp. 1210-1218, 2010. [Online]: https://doi.org/10.1111/j.15396924.2010.01428.x

8. European Centre for Disease Prevention and Control, "Using face masks in the community: first update - Effectiveness in reducing transmission of COVID-19," 2021 [Online]:

https://www.ecdc.europa.eu/en/publications-data/using-face-maskscommunity-reducing-covid-19-transmission

9. Mills, M., Rahal, C., and Akimova, E., "Face masks and coverings for the general public: behavioural knowledge, effectiveness of cloth coverings and public messaging," . The Royal Society \& The British Academy, 2020. [Online]: https://royalsociety.org//media/policy/projects/set-c/set-c-facemasks.pdf

10. Bunyan, D., Ritchie, L., Jenkins, D., and Coia, J. E., "Respiratory and facial protection: a critical review of recent literature," J. Hosp. Infect., vol. 85 , no. 3, pp. 165-169, 2013. [Online]: https://doi.org/10.1016/j.jhin.2013.07.011

11. Leung, N. H., Chu, D. K., Shiu, E. Y., Chan, K. H., McDevitt, J. J., Hau, B. J., Yen, H. L., Li, Y., Ip, D. K., Peiris, J. S., Seto, W. H., Leung, G. M., Milton, D. K., and Cowling, B. J., "Respiratory virus shedding in exhaled breath and efficacy of face masks," Nat. med., vol. 26, no. 5, pp. 676-680, 2020. [Online]: https://doi.org/10.1038/s41591-020-0843-2

12. US Food and Drug Administration, "N95 Respirators, Surgical Masks, and Face Masks.” [Online]: https://www.fda.gov/medicaldevices/personal-protective-equipment-infection-control/n95respirators-surgical-masks-and-face-masks

13. Gralton, J. and McLaws, M. L., "Protecting healthcare workers from pandemic influenza: N95 or surgical masks?," Crit. Care Med., vol. 38, no. 2, pp. 657-667, 2010. [Online]: https://doi.org/10.1097/CCM.0b013e3181b9e8b3

14. Liao, L., Xiao, W., Zhao, M., Yu, X., Wang, H., Wang, Q., Chu, S., and Cui, Y., "Can N95 respirators be reused after disinfection? How many times?," ACS nano, vol. 14, no. 5, pp. 6348-6356, 2020. [Online]: https://doi.org/10.1021/acsnano.0c03597

15. Wang, D., Sun, B. C., Wang, J. X., Zhou, Y. Y., Chen, Z. W., Fang, Y., Yue, W. H., Liu, S. M., Liu, K. Y., Zeng, X. F., and Chen, J. F., "Can masks be reused after hot water decontamination during the COVID-19 pandemic?," Engineering, vol. 6, no. 10, pp. 1115-1121, 2020. [Online]: https://doi.org/10.1016/j.eng.2020.05.016

16. Swennen, G. R., Pottel, L., and Haers, P. E., "Custom-made 3D-printed face masks in case of pandemic crisis situations with a lack of commercially available FFP2/3 masks," Oral Maxil. Surg., vol, 49, no.
5, pp. 673-677, 2020. [Online]:

https://doi.org/10.1016/j.ijom.2020.03.015

17. Tian, S., Yang, W., Le Grange, J. M., Wang, P., Huang, W., and Ye, Z., "Smart healthcare: making medical care more intelligent," Global

Health Journal, vol. 3, no. 3, pp. 62-65, 2019. [Online]: https://doi.org/10.1016/i.glohj.2019.07.001

18. Baker, S. B., Xiang, W., and Atkinson, I., "Internet of things for smart healthcare: Technologies, challenges, and opportunities," IEEE Access, vol. 5, pp. 26521-26544, 2017. [Online] https://doi.org/10.1109/ACCESS.2017.2775180

19. Cheung, M. L., Leung, K. S., and Chan, H. S., "Driving healthcare wearable technology adoption for Generation $\mathrm{Z}$ consumers in Hong Kong." Young Consumers, 2020. [Online]: https://doi.org/10.1108/YC04-2020-1123

20. Cheung, M. L., Chau, K. Y., Lam, M. H. S., Tse, G., Ho, K. Y., Flint, S. W., Broom, D. R., Tso, E. K., and Lee, K. Y., 'Examining consumers' adoption of wearable healthcare technology: The role of health attributes," Int. J. Environ. Res. Public Health, vol. 16, no. 13, p. 11-16, 2019. [Online]: https://doi.org/10.3390/ijerph16132257

21. Myers, B., Nahal, J. A., Yang, C., Brown, L., Ghiasi, S., and Knoesen, A., "Towards data-driven pre-operative evaluation of lung cancer patients: the case of smart mask," In 2016 IEEE Wireless Health (WH), 2016, pp. 1-6. [Online]: https://doi.org/10.1109/WH.2016.7764569

22. Tripp Jr, L. D., and Albery, W. B., "Development of an Oxygen Mask Integrated Arterial Oxygen Saturation (SaO2) Monitoring System for Pilot Protection in Advanced Fighter Aircraft," Harry G Armstrong Aerospace Medical Research Lab Wright-Patterson AFB OH, 1987. [Online]: https://apps.dtic.mil/sti/pdfs/ADA323229.pdf

23. Cheng, Y., Wang, C., Zhong, J., Lin, S., Xiao, Y., Zhong, Q., Jiang, H., Wu, N., Li, W., Chen, S., Wang, B., Zhang, Y., and Zhou, J., "Electrospun polyetherimide electret nonwoven for bi-functional smart face mask," Nano Energy, vol. 34, pp. 562-569, 2017. [Online]: https://doi.org/10.1016/j.nanoen.2017.03.011

24. Ghatak, B., Banerjee, S., Ali, S. B., Bandyopadhyay, R., Das, N., Mandal, D., and Tudu, B., "Design of a Self-powered Smart Mask for COVID-19," 2020. arXiv preprint arXiv:2005.08305. [Online]: https://arxiv.org/abs/2005.08305

25. Pan, L., Wang, C., Jin, H., Li, J., Yang, L., Zheng, Y., Wen, Y., Tan, B. H., Loh, X. J., and Chen, X., "Lab-on-Mask for Remote Respiratory Monitoring," ACS Mater. Lett., vol. 2, no. 9, pp. 1178-1181, 2020. [Online]: https://doi.org/10.1021/acsmaterialslett.0c00299

26. Kalavakonda, R. R., Masna, N. V. R., Bhuniaroy, A., Mandal, S., and Bhunia, S.,"A Smart Mask for Active Defense Against Coronaviruses and Other Airborne Pathogens," IEEE Consumer Electronics Magazine, vol. 10, no. 2, pp. 72-79, 2020. [Online]: https://doi.org/10.1109/MCE.2020.3033270

27. The World's Smartest Mask - Project Hazel - Razer. [Online]: https://www.razer.com/concepts/razer-project-hazel

28. Active + Halo Smart Mask - AirPop. [Online]: https://www.airpophealth.com/us/airpop-active-smart-black-yellow

29. Silva, A. L. P., Prata, J. C., Walker, T. R., Duarte, A. C., Ouyang, W., Barcelò, D., and Rocha-Santos, T., "Increased plastic pollution due to COVID-19 pandemic: Challenges and recommendations," Chem. Eng. $J$. , vol. 405, no. $1,126683,2021$. [Online]: https://doi.org/10.1016/i.cej.2020.126683

30. Shi, W. and Dustdar, S., "The promise of edge computing," Computer, vol. 49, no. 5, pp. 78-81, 2016. [Online] https://doi.org/10.1109/MC.2016.145 